\title{
Escala de Percepçáo de Suporte Organizacional: evidência da estrutura fatorial em trabalhadores brasileiros
}

\author{
Organizational Support Perception Scale: evidence of factorial structure in Brazilian workers
}

\author{
Luis Felipe de Oliveira Fleury ${ }^{\mathrm{I}}$ \\ Nilton Soares Formiga ${ }^{\mathrm{II}}$ \\ Marcos Aguiar de Souza ${ }^{\text {III }}$ \\ Marco Antônio Ferreira de Souza ${ }^{\text {IV }}$
}

\section{Resumo}

As crenças e as expectativas do indivíduo acerca da retribuição e do reconhecimento dado pela organizaçáo ao seu trabalho são questóes diretamente relacionadas à percepçáo de suporte organizacional. No presente estudo pretendeu-se investigar evidências psicométricas da Escala de Percepçáo de Suporte Organizacional (EPSO) em trabalhadores brasileiros. Para isso foram coletados dados com 635 pessoas. Foi realizado um procedimento de análise fatorial exploratória que demonstrou índices satisfatórios $(\mathrm{KMO}=0,85 ; \mathrm{p}<0,001)$ e apontou o instrumento com um único fator que explica $49,9 \%$ da variância. O procedimento de análise fatorial confirmatória com o auxílio do software AMOS apontou índices satisfatórios de adequação do modelo. Os resultados reforçam a solução unifatorial representada de acordo com o que se esperava teoricamente.

Palavras chave: suporte organizacional; análise fatorial confirmatória; validação.

\section{Abstract}

The perception of organizational support is related to the belief that the company values the work developed by the worker. This study aimed to investigate the psychometric evidence of the Organizational Support Perception Scale in Brazilian workers. To meet this goal were collected data with 635 people. An exploratory factor analysis procedure was performed that showed satisfactory indexes $(\mathrm{KMO}=0.85 ; \mathrm{p}<0.001)$ and pointed the instrument with a single factor that explains $49,9 \%$ of the variance. The procedure of confirmatory factor analysis was made with the aid of the AMOS software and satisfactory indexes of adequacy of the model were found. The results reinforce the one-way solution represented according to what was theoretically expected.

Keywords: Organizational support; confirmatory factor analysis; validation.

\footnotetext{
${ }^{\mathrm{I}}$ Mestrando em Psicologia UFRJ, Universidade Federal do Rio de Janeiro

II Pós - Doutorado em Psicologia pela UFRRJ, Faculdade Internacional da Paraíba

III Professor do Programa de Pós - Graduaçáo em Psicologia da Universidade Federal do Rio de Janeiro

${ }^{\text {IV }}$ Professor da Departamento de Ciências Administrativas e Contábeis da UFRRJ, Universidade Federal Rural do Rio de Janeiro
}

Os estudos envolvendo a dinâmica organizacional devotam preocupaçáo constante com a maneira como os indivíduos experimentam e definem a realidade dentro das organizaçôes. Especificamente, a área da ciência humana e social aborda a preocupação dos gestores com as quedas de produtividade relacionando-as as condiçóes de administração dos recursos humanos na organização. As evidencias teóricas e empíricas reforçam há muito que o sucesso no alcance dos objetivos e metas organizacionais depende da existência de um grupo de trabalhadores comprometidos e identificados com a instituição (Brandão, Borges-Andrade \& Guimarães, 2012).

Nos últimos anos, as pesquisas procuraram explorar os efeitos na organizaçáo decorrentes do absenteísmo (Oenning, Carvalho \& Lima, 2012), da intenção de rotatividade (Agapito, Polizzi Filho \& Siqueira, 2015), da baixa qualidade de vida no trabalho (Sant'Anna \& Kilimnik, 2011), motivação (Tamayo \& Paschoal, 2003), entre outras variáveis relacionadas ao corpo de trabalhadores.
Esse conjunto de investigaçóes compóe um esforço da comunidade acadêmica de oferecer contribuiçóes para lidar com tais problemas na prática intraorganizacional.

Reconhece-se cada vez mais a importância de a organização oferecer aos seus colaboradores recursos que permitam que os mesmos desenvolvam seus respectivos trabalhos da melhor forma possível evitando, assim, os problemas das situaçóes supracitadas. As organizaçóes preocupam-se, então, em disponibilizar, por exemplo, incentivo à capacitação, gratificaçóes por desempenho, auxílio creche, entre outros, que são compreendidos como recursos de suporte organizacional. As pesquisas na área da Psicologia Organizacional e do Trabalho indicam que uma percepção de suporte organizacional elevada está associada a aspectos positivos do trabalhador na organização, tais como comprometimento, motivação, qualidade de vida (Kalidass \& Bahron, 2015; Formiga, Fleury \& Souza, 2014; Brandão et al., 2012; Paschoal, 2008; Oliveira-Castro, Pilati \& Borges-Andrade, 1999). 
Os estudos pioneiros a respeito da percepção de suporte organizacional são de Eisenberger, Huntington, Hutchison e Sowa (1986). Os autores colocam que a percepção de suporte organizacional tem relação com crenças globais desenvolvidas pelo empregado sobre a extensão em que a organização valoriza as suas contribuiçóes e cuida do seu bem-estar.

Oliveira-Castro et al. (1999) afirmam que a literatura especializada acerca do tema, vem mostrando a existência de correlaçóes positivas entre percepção de suporte organizacional e medidas de desempenho no trabalho, comportamentos de cidadania organizacional, criatividade e inovaçáo, etc. Sendo assim, o suporte organizacional seria uma variável preditora de outras variáveis no âmbito organizacional.

Para Jawahar e Hemmasi (2006) a percepção de suporte organizacional se desenvolve através das múltiplas situaçóes de troca existentes entre o empregado e o empregador/empresa. Ou seja, o suporte organizacional é uma forma de contrato psicológico baseado em expectativas de troca e benefícios mútuos que são estabelecidos pelo trabalhador com a sua organização (Paschoal, 2008).

Eisenberger et al. (1986), em estudo clássico na área, propuseram um instrumento para mensurar a percepção de suporte organizacional. $\mathrm{O}$ instrumento intitulado 'Survey of Perceived Organizational Support', desenvolvido pelos autores, em sua forma completa, é composto por 36 itens e 17 itens em formato reduzido. É válido ressaltar que a escala na sua versão completa é dividida, metade dos itens sáo positivos e a outra metade, negativos.

No contexto brasileiro destaca-se a versão reduzida da Escala de Percepção de Suporte Organizacional validada por Siqueira (1995) em seu estudo sobre comportamentos de cidadania organizacional, adaptando o instrumento de Eisenberger et al. (1986) às características culturais brasileiras numa amostra de 287 trabalhadores. Por fim, Oliveira-Castro et al. (1999) também propuseram um instrumento para mensuração da variável no contexto brasileiro.

Tanto o estudo de Eisenberger et al. (1986), os de Siqueira $(1995 ; 2008)$ quanto o de Oliveira-Castro et al. (1999) sugerem que o trabalhador formula opinióes globais sobre o quanto a organização valoriza suas contribuiçôes e cuida do seu bem-estar. Essas opinióes referem-se às crenças do trabalhador sobre o comportamento da organização como um todo e não sobre o comportamento de agentes organizacionais específicos como os de chefes e líderes.

Kalidass e Bahron (2015) investigaram a influência da percepção de suporte organizacional na intenção de rotatividade entre trabalhadores do setor hoteleiro na Malásia. Os resultados encontrados pelos autores demonstram uma associação significativa negativa entre ambas as variáveis. Os pesquisadores apontam em seu estudo que uma baixa percepção, por parte dos trabalhadores, que a organização se preocupa com eles, influi significativamente na intenção destes trabalhadores de sair da empresa.

O presente estudo tem dois objetivos principais: 1 - com uma primeira amostra, a partir de uma análise fatorial exploratória pretendeu-se conhecer a distribuição item-fator e fidedignidade da Escala de Percepção de Suporte Organizacional. Entende-se esta análise como um conjunto de técnicas multivariadas que tem como objetivo encontrar a estrutura subjacente em uma matriz de dados e determinar o número e a natureza das variáveis latentes (fatores) que melhor representam um conjunto de variáveis observadas (Damásio, 2012). 2 - Ainda que no primeiro estudo tenha sido observada uma relação itemfator confiável, o cálculo realizado se baseia estritamente nos dados obtidos e não considera um modelo teórico fixo que oriente a extração das dimensões latentes. Por esta razão, teria pouco poder de apresentar qualquer indicação sobre o bom ajustamento do modelo para futuros estudos. A fim de propor uma resolução para tal limitação do primeiro estudo, com uma segunda amostra, foi realizada uma análise fatorial confirmatória.

A técnica da análise fatorial confirmatória, em síntese, visa indicar a adequação do modelo empírico com base na teoria. A técnica tem a clara vantagem de considerar, justamente, a teoria para definir os itens pertencentes a cada fator, bem como, apresentar indicadores de adequação de ajuste que permitem decidir objetivamente sobre a validade de construto da medida analisada em comparação com as outras escalas exploradas (Damásio, 2013).

\section{Método}

\section{Participantes}

Participaram do estudo 635 trabalhadores, com idade variando entre 18 e 73 anos e média de 45,79 anos, $45 \%$ de instituiçóes públicas e $55 \%$ de instituiçôes privadas, $52,8 \%$ eram sujeitos do sexo masculino e $54 \%$ solteiros.

Para atender aos objetivos, o banco de dados foi dividido em duas amostras entre participantes pares e ímpares de acordo com a ordem que apareciam no banco de dados: a primeira com 318 sujeitos e a segunda com 317 sujeitos. As amostras foram do tipo intencional, pois 
considerou-se a pessoa que, consultada, se dispusera a colaborar respondendo o questionário.

\section{Instrumentos}

Escala de Percepção de Suporte Organizacional (EPSO): trata-se de um instrumento desenvolvido por Eisenberger et al. (1986). No Brasil, Siqueira (1995; 2008) realizou estudos de validação e adaptação do instrumento.

A presente escala, de acordo com Siqueira (1995; 2008), é unifatorial, composta por 9 itens, que explicaram 55\% da variância no estudo da referida autora. A autora observou correlações itens-fator acima de $0,40 \mathrm{e}$ um alfa $(\alpha)$ de Cronbach de 0,86 . Seguindo a direção teórico-metodológica, proposta pela autora, Formiga et al. (2014), realizaram com 490 trabalhadores de diferentes empresas uma análise fatorial confirmatória e avaliaram a estrutura fatorial da escala tendo observado indicadores psicométricos que ratificaram a organização item-fator da escala em questáo.

\section{Procedimentos Éticos}

Todos os procedimentos adotados nesta pesquisa seguiram as orientações previstas na Resolução 196/96 do Conselho Nacional de Saúde (1996) e da Resolução 016/2000 do Conselho Federal de Psicologia (2000).

Colaboradores com experiência prévia na administração de instrumentos em Psicologia foram responsáveis pela coleta dos dados, e apresentaram-se nas empresas como interessados em conhecer as opinióes e os comportamentos dos colaboradores sobre as situaçóes descritas na escala.

Solicitou-se a colaboração voluntária das pessoas no sentido de responderem um breve questionário. Após ficarem cientes das condiçóes de participaçáo na pesquisa, assinaram um termo de Consentimento Livre e Esclarecido. Foi-lhes dito que não havia resposta certa ou errada. A todos foi assegurado o anonimato das suas respostas informando que estas seriam tratadas em seu conjunto. A EPSO foi respondida individualmente ou em grupo em organizaçóes públicas e privadas no estado do Rio de Janeiro. E os participantes puderam interromper a qualquer momento a participação.

\section{Análise dos Dados}

Com a finalidade de atender aos objetivos descritos na introdução, a análise de dados foi realizada em duas etapas:
$\mathrm{Na}$ etapa 1, com 318 sujeitos, os dados foram analisados através do software SPSS (versão 21.0). Além de estatísticas descritivas (média, desvio padrão, frequência), realizou-se uma análise de Componentes Principais $(\mathrm{CP})$, não estabelecendo rotação, pois, com base nos estudos anteriores a hipótese é de um instrumento unifatorial.

Posteriormente, calculou-se a consistência interna (Alfa de Cronbach) do fator resultante. Contudo, previamente, considerou-se a própria possibilidade de se realizar a análise dos Componentes Principais, tomando como critérios o KMO igual ou superior a 0,70 e o Teste de Esfericidade de Bartlett (qui-quadrado, $\chi^{2}$ ) significativo $(p<0,05)$ (Tabachnick \& Fidell, 2001; Bisquerra, 1989; Dancey \& Reidy, 2006). Além de considerar que os critérios de Kaiser (valor próprio igual ou superior a 1) e Cattell (distribuição gráfica dos valores próprios, visando distinguir aqueles sobressalentes) tendem a maximizar o número de fatores a extrair decidiu-se, para isso, efetuar uma Análise Paralela, indicada pela literatura especializada (Bisquerra, 1989; Dancey \& Reidy, 2006; Hayton, Allen \& Scarpello, 2004; Ledesma \& Valero-Mora, 2007). Neste caso, teve-se em conta a sintaxe do SPSS desenvolvida por O'Connor (2000) a fim de realizar a Análise Paralela proposta.

$\mathrm{Na}$ etapa 2, com 317 sujeitos, utilizou-se para a análise dos dados a versão 21.0 do pacote estatístico SPSS para as análises descritivas e para a análise fatorial confirmatória, utilizou-se o programa AMOS 18.0. Esse programa estatístico tem a função de apresentar, de forma mais robusta, indicadores psicométricos que visam uma melhor construção da adaptação e acurácia da escala estudada, bem como, permite desenhar o modelo teórico pretendido no estudo.

Pretendeu-se, então, testar a adequação do modelo unidimensional, considerando como entrada a matriz de covariâncias, tendo sido adotado o estimador $M L$ (Maximum Likelihood). Este tipo de análise estatística é mais criteriosa e rigorosa do que aquela realizada no primeiro estudo apresentado. Isto permite testar diretamente uma estrutura teórica, como é o caso da que se propóem no presente estudo.

Esta análise apresenta alguns índices que permitem avaliar a qualidade de ajuste do modelo proposto, esses índices são recomendados e discutidos na literatura (Byrne, 1989; Hair, Anderson, Tatham \& Black, 2005; Kelloway, 1998; Tabachnick \& Fidell, 1996; Van de Vijver \& Leung, 1997), por exemplo:

O qui-quadrado $\left(\chi^{2}\right)$ testa a probabilidade de o modelo teórico se ajustar aos dados, ou seja, quanto maior este valor pior o ajustamento. Este tem sido pouco 
empregado na literatura, sendo mais comum considerar sua razão em relação aos graus de liberdade $\left(\chi^{2} /\right.$ g.l). Neste caso, valores até 5 indicam um ajustamento adequado.

O índice de Raiz Quadrada Média Residual (RMR), que indica o ajustamento do modelo teórico aos dados, na medida em que a diferença entre os dois se aproxima de zero.

Comparative Fit Index (CFI) que compara de forma geral o modelo estimado e o modelo nulo, considerando valores mais próximos de um como indicadores de ajustamento satisfatório.

Tucker-Lewis Index (TLI), apresenta uma medida de parcimônia entre os índices do modelo proposto e do modelo nulo. Varia de zero a um, com índice aceitável acima de 0,90 .

Goodness-of-Fit Index (GFI) e o Adjusted Goodnessof-Fit Index (AGFI) são análogos ao $\mathrm{R}^{2}$ em regressão múltipla. Portanto, indicam a proporçáo de variânciacovariância nos dados explicada pelo modelo. Estes variam de 0 a 1 , com valores na casa dos 0,80 e 0,90 , ou superior, indicando um ajustamento satisfatório.

Root-Mean-Square Error of Approximation (RMSEA), com seu intervalo de confiança de 90\% (IC90\%), é considerado um indicador de desajustamento do modelo proposto, isto é, valores altos indicam um modelo não ajustado. Assume-se como ideal que o RMSEA se situe entre 0,05 e 0,08, aceitando-se valores de até 0,10.

\section{Resultados}

\section{Etapa 1}

Buscou-se avaliar, a partir dos pressupostos da Teoria Clássica dos Testes (TCT), se os itens apresentavam capacidade de discriminar pessoas com magnitudes próximas, ou seja, discriminar aquelas dos grupos inferiores e superiores com relação ao construto medido (Pasquali, 2011). Outro motivo para realizar este cálculo se deve por não encontrar esta análise nos estudos supracitados sobre o tema (ver Eisenberger et al., 1986; Siqueira, 1995; 2008; Formiga et al., 2014).

Com a finalidade de observar a discriminação dos itens da escala, foi calculada uma pontuação total da escala e em seguida sua mediana. Os respondentes com pontuação abaixo da mediana foram classificados como sendo do grupo inferior, enquanto que aquelas com pontuaçóes acima da mediana foram definidas como do grupo superior. Considerando-se cada um dos itens desta medida, efetuou-se um teste $t$ para amostras independentes e compararam-se ambos os grupos observando quais dos itens da escala foram capazes de discriminar pessoas com magnitudes próximas estatisticamente significativas (ver Tabela 1). Estes itens foram retidos na escala, justificando sua inserção numa posterior análise fatorial dos principais componentes.

\section{Tabela 1}

Poder discriminativo dos itens da Escala de Percepção de Suporte Organizacional

\begin{tabular}{|c|c|c|c|}
\hline Itens & $t$ & $r$ (pontuaçáo total) & $\mathbf{p}<$ \\
\hline 1. Esta organização ignoraria qualquer reclamação de minha parte. & $-10,17$ & 0,64 & 0,01 \\
\hline $\begin{array}{c}\text { 2. Essa organização não considera meus interesses } \\
\text { quando toma decisões que me afetam. }\end{array}$ & $-, 8,40$ & 0,61 & 0,01 \\
\hline $\begin{array}{l}\text { 3. É possível obter ajuda desta organização } \\
\text { quando tenho um problema. }\end{array}$ & $-12,42$ & 0,60 & 0,01 \\
\hline 4. Esta organização realmente preocupa-se com o meu bem-estar. & $-16,46$ & 0,83 & 0,01 \\
\hline $\begin{array}{l}\text { 5. Esta organização estaria disposta a ampliar suas } \\
\text { instalaçóes para me ajudar a utilizar minhas melhores } \\
\text { habilidades no desempenho do meu trabalho. }\end{array}$ & $-11,47$ & 0,72 & 0,01 \\
\hline $\begin{array}{l}\text { 6. Esta organizaçáo esta pronta a ajudar-me } \\
\text { quando eu precisar de um favor especial. }\end{array}$ & $-15,55$ & 0,80 & 0,01 \\
\hline 7. Esta organização preocupa-se com a minha satisfação no trabalho. & $-14,33$ & 0,80 & 0,01 \\
\hline $\begin{array}{c}\text { 8. Esta organização preocupa-se mais com os } \\
\text { seus lucros (ganhos) do que comigo. }\end{array}$ & $-7,59$ & 0,56 & 0,01 \\
\hline $\begin{array}{l}\text { 9. Esta organização tenta fazer com que o meu } \\
\text { trabalho seja o mais interessante possível. }\end{array}$ & $-11,01$ & 0,74 & 0,01 \\
\hline
\end{tabular}

Na Tabela 1, é possível observar que os itens da Escala de Percepção Suporte Organizacional, discriminam uma espécie de contrato psicológico capaz de gerar expectativas de troca e benefícios entre empregado e empregador e/ou gestor na sua organizaçáo. Optou-se também por avaliar a relação do conteúdo dos itens, tendo como objetivo verificar a representatividade comportamento-domínio, a qual, sistematicamente, avalia a 
relação teórica apresentada no instrumento de medida, desenvolvido por Eisenberger et al. (1986) e adaptado por Siqueira (1995), para o contexto brasileiro e as situaçóes especificadas nos itens. Ou seja, o quanto este instrumento representa os aspectos esperados (Cunha, 2000; Pasquali, 2011).

A partir dessa perspectiva, efetuou-se uma análise de correlação linear de Pearson (r) para a referida escala, destinada à avaliação dos itens referentes ao desenvolvimento do suporte organizacional em trabalhadores, nestes, teoricamente, foram contemplados os itens propostos pelos autores supracitados. Ainda na Tabela 1, é possível observar que todas as correlaçôes relativas aos itens relacionados à percepção de suporte organizacional (pontuação total da escala), foram significativamente positivas.

Observados esses resultados, decidiu-se efetuar uma análise de Componentes Principais, sem fixar o número de fatores a extrair e sem rotação, assumindo uma saturação de $\pm 0,30$. Visando a segurança na tomada de decisão na escolha dos fatores, três critérios foram levados em conta: (1) quantidade de valores próprios (eigenvalues) iguais ou superiores a 1 (Critério de Kaiser), (2) distribuição gráfica dos valores próprios, tomando como referência o ponto a partir do qual nenhum outro fator aporta consideravelmente para a estrutura (Critério de Cattell) e (3) Análise Paralela (Bisquerra,1989; Fabrigar, Wegerer, MacCallum \& Strahan, 1999; O'Connor, 2000; Hayton et al., 2004; Dancey \& Reidy, 2006; Ledesman \& Valero-Mora, 2007). Os resultados das análises permitiram identificar a adequação da matriz de correlação: $\mathrm{KMO}=0,85$ e do Teste de Esfericidade de Bartlett, $\chi^{2} / \mathrm{gl}=1393,11 / 36, \mathrm{p}<0,001$. Na distribuição gráfica, os valores próprios (critério de Cattell), demonstraram a bifatorialidade da escala (ver Figura 1).

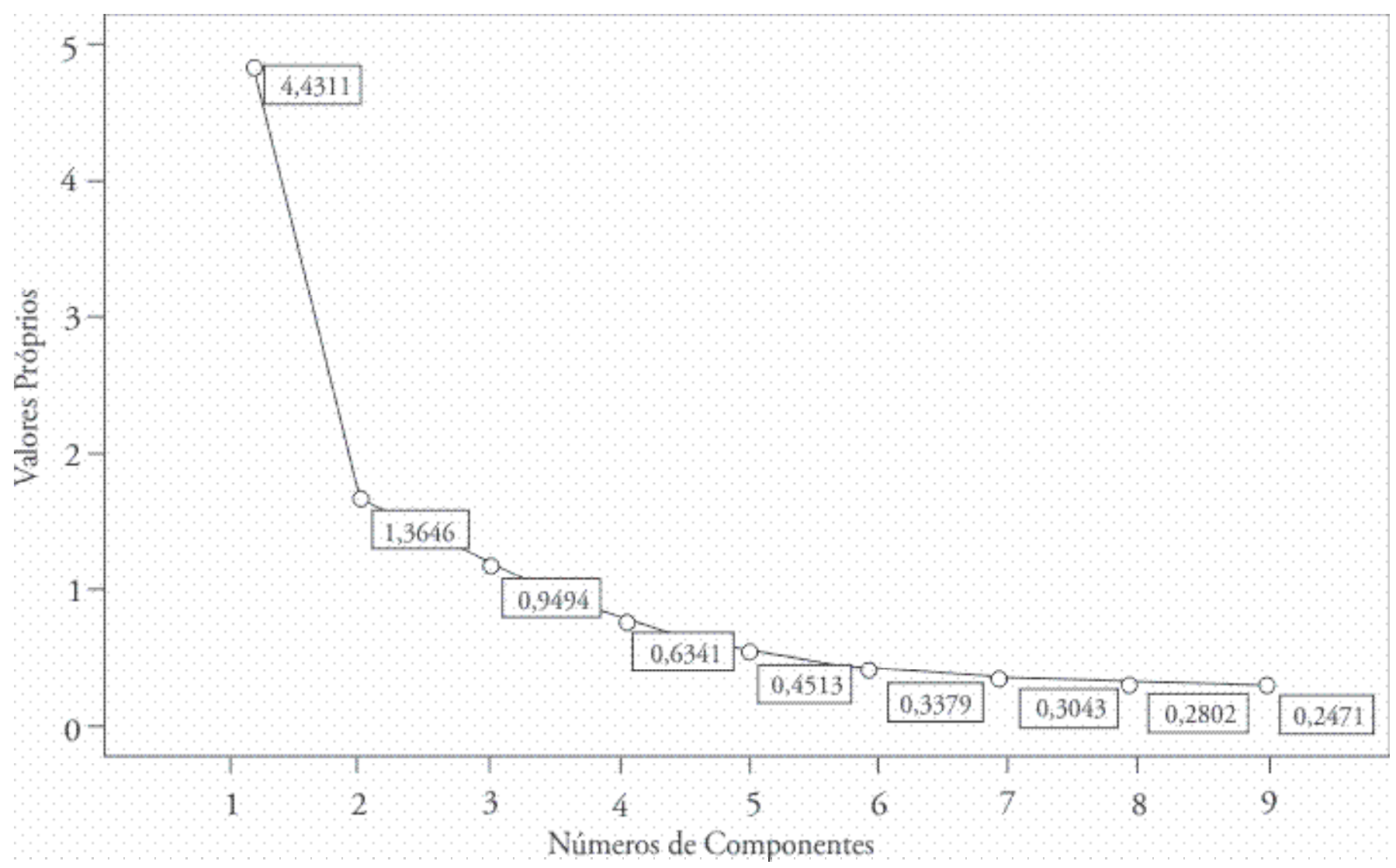

Figura 1

Diagrama de declividade do número de fatores plotados sobre a percep̧̧ão de suporte organizacional

De acordo com o critério de Kaiser (isto é, os valores próprios - Eigenvalues), identificou-se também, a existência de dois fatores com valores próprios maiores que 1 (um), explicando conjuntamente $64,40 \%$ da variância total. Alternativamente, procurando não deixar dúvidas quando a dimensionalidade do construto, realizou-se a Análise Paralela, assumindo os mesmos parâmetros do banco de dados original. Isto é, 318 participantes e 9 (nove) variáveis, tendo seus valores próprios gerados em 1.000 simulaçôes aleatórias com os itens (ver Tabela 2). 


\section{Tabela 2}

Distribuição dos fatores da análise de Componentes Principais de acordo com o critério de Kaiser e Análise Paralela

\begin{tabular}{cc}
\hline Critério de Kaiser & Análise Paralela \\
\hline $\mathbf{4 , 4 3}$ & $\mathbf{1 , 2 6}$ \\
1,37 & 1,48 \\
\hline
\end{tabular}

Contrastando estes valores com aqueles observados empiricamente e com os valores próprios, isto é, entre os critérios de Kaiser e Análise Paralela, o critério de Kaiser apresentou valores superiores aos simulados, permitindo assim, identificar um único fator pertencente à escala em questáo. Atento a interpretaçáo do item-fator, juízes especializados nas análises efetuadas, contribuíram com seu julgamento, corroborando para a decisão que se esperava. Assim, fixou-se a Escala de Percepção de Suporte Organizacional como uma medida unifatorial estando amparados pelas recomendaçóes da literatura especializada (Hayton et al., 2004).
A partir desses critérios estabelecidos procedeu-se a realização de uma análise dos Componentes Principais sem rotação, por considerar, empiricamente, ser instrumento unifatorial. Considerou-se um eigenvalue (valor próprio) $\geq 1,00$ para definir o fator e saturação de $\pm 0,30$ para retenção dos itens. A utilização desta técnica se mostrou bastante adequada através do $K M O=0,90$ e do Teste de Esfericidade de Bartlett, $\chi^{2} / \mathrm{gl}=1386,44 / 28, p<0,001$.

Os resultados da extração revelaram a presença de um único fator principal, refutando a bifatorialidade, de acordo com o encontrado nas análises para tomada de decisão, sendo: valores próprios (eigenvalues) iguais ou superiores a 1 (Critério de Kaiser), distribuição gráfica dos valores próprios (Critério de Cattell) e a Análise Paralela. Este fator explicou 49,99\% da variância total (ver Tabela 3). Para facilitar a compreensão do leitor, apresentou-se o conteúdo de cada item, sua saturaçáo (carga fatorial) e comunalidade, bem como, os indicadores de consistência interna (Alfa de Cronbach) e variância explicada.

\section{Tabela 3}

\section{Análise dos Componentes Principais da Escala de Percepção de Suporte Organizacional}

\begin{tabular}{ccc}
\hline Itens & $\mathbf{a}_{\text {i.f }}$ & $\mathbf{h}^{\mathbf{2}}$ \\
\hline 1. Esta organização ignoraria qualquer reclamação de minha parte. & 0,84 & 0,76 \\
2. Essa organização não considera meus interesses quando toma decisões que me afetam. & 0,83 & 0,71 \\
3. É possível obter ajuda desta organização quando tenho um problema. & 0,82 & 0,44 \\
4. Esta organização realmente preocupa-se com o meu bem-estar. & 0,77 & 0,73 \\
5. Esta organização estaria disposta a ampliar suas instalações para me ajudar a & 0,75 & 0,64 \\
utilizar minhas melhores habilidades no desempenho do meu trabalho. & 0,60 & 0,76 \\
6. Esta organização esta pronta a ajudar-me quando eu precisar de um favor especial. & $0,600.70$ \\
7. Esta organização preocupa-se com a minha satisfação no trabalho. & 0,59 & 0.43 \\
8. Esta organização preocupa-se mais com os seus lucros (ganhos) do que comigo. & $0,360.62$ \\
9. Esta organização tenta fazer com que o meu trabalho seja o mais interessante possível. & 09 \\
\hline Número total de itens & 09,43 \\
Valores Próprios & 49,99 \\
Variância Explicada & 0,86 \\
\hline
\end{tabular}

Notas. $\mathrm{a}_{\mathrm{if}}=$ Carga Fatorial; $\mathrm{h}^{2}=$ Cumunalidade. $\mathrm{N}=318$ sujeitos.

Considerando esses resultados, pode-se destacar, na análise fatorial exploratória da escala, sua estrutura unifatorial sendo possível afirmar que a sua organização fatorial foi consistente. Porém, como na análise exploratória, a sua aleatoriedade não permite teorizar sobre as variáveis destacadas neste estudo, em um segundo momento, tratou-se de realizar uma análise fatorial confirmatória.
Para tal, utilizou-se o programa AMOS 18.0, destinado ao cálculo de modelagem de equaçôes estruturais e análise fatorial confirmatória. Esse programa estatístico tem a funçáo de apresentar, de forma mais robusta, indicadores psicométricos que visam uma melhor construção da adaptaçáo e acurácia da escala avaliada anteriormente, bem como, permite desenhar um modelo teórico que se pretende. 


\section{Etapa 2}

A amostra contou com 317 sujeitos com características semelhantes aos da etapa 1 . Optou-se por deixar livre a covariância (phi, $\phi$ ) entre os fatores. Os indicadores de qualidade de ajuste do modelo se mostraram próximos aos recomendados na literatura (Byrne, 1989; Tabachnick \& Fidell, 1996; van de Vijver \& Leung,
1997). Os resultados desta análise podem ser observados, a partir da seguinte razão: $\chi^{2} / \mathrm{gl}(34,35 / 23)=1,56$, $\mathrm{RMR}=0,04, G F I=0,98, \mathrm{AGFI}=0,95, C F I=0,99$, $\mathrm{TLI}=0,99$, RMSEA $(90 \% \mathrm{IC})=0,04(0,01-0,07) . \mathrm{O}$ valor do $E C V I$ e $C A I C$ não foram necessários por não haver modelos para serem comparados. Na Tabela 4 é apresentada a estrutura fatorial resultante (solução padronizada) dessa análise.

\section{Tabela 4}

Estrutura fatorial da Escala de Percepção de Suporte Organizacional

\begin{tabular}{cccccc}
\hline $\boldsymbol{\Xi}$ (construto) & $\boldsymbol{\chi}$ (variáveis) [itens] & $\boldsymbol{\Lambda}$ (carga fatorial) & $\boldsymbol{\varepsilon}$ (erros) & CC & VME \\
\hline & SO1 & 0,71 & 0,50 & & \\
& SO2 & 0,69 & 0,48 & & \\
SO3 & 0,65 & 0,42 & & \\
Percepção de & SO4 & 0,85 & 0,73 & & \\
Suporte & SO5 & 0,78 & 0,60 & 0,56 \\
Organizacional & SO6 & 0,72 & 0,53 & & \\
& SO7 & 0,85 & 0,73 & & \\
& SO8 & 0,51 & 0,37 & & \\
& SO9 & 0,81 & 0,65 & & \\
\hline
\end{tabular}

Notas. SO1, SO2, SO3... = itens da escala; CC = Confiabilidade Composta; VME = Variância Média Extraída

Como é possível observar, todas as saturaçóes (Lambdas, $\lambda$ ) estão dentro do intervalo esperado $\mid 0$ - $1 \mid$, denotando não haver problemas de estimação proposta. Além disso, todas são estatisticamente diferentes de zero $(t>1,96, p<0,05)$, corroborando a existência unifatorial da medida verificada no primeiro estudo realizado a partir da análise fatorial exploratória.

É necessário salientar, no que se refere à validade deste construto, que foi realizado, também, tanto o cálculo de Confiabilidade Composta (CC) quanto o cálculo da Variância Média Extraída (VME) nos termos recomendados pela literatura (Valentini \& Damásio, 2016).
No primeiro indicador exige-se que o nível do escore seja acima de 0,70 , enquanto no segundo indicador é preciso um nível acima de 0,50 .

Observou-se que para a dimensão da escala, a CC e a VME, estiveram acima do exigido na literatura, respectivamente, 0,92 e 0,56 , condiçáo que evidencia a confiabilidade e validade convergente do construto representado. É possível observar que tal resultado é confirmado pela observação das estimativas de predição a partir da análise de regressão para tal modelo, as quais identificam as variáveis como significativas e com uma razão critério dentro do que é estatisticamente exigido (ver Tabela 5).

Tabela 5

Estimativas de predição a partir da análise de regressão

\begin{tabular}{ccccccc}
\hline Itens & - & Fator & Estimativa & $\begin{array}{c}\text { Desvio } \\
\text { Padráo }\end{array}$ & $\begin{array}{c}\text { Razáo } \\
\text { Critério }\end{array}$ & p $<$ \\
\hline SO9 & $<---$ & SOrg & 1,000 & -- & -- & -- \\
SO8 & $<---$ & SOrg &, 677 &, 072 & 9,408 & 0,01 \\
SO7 & $<---$ & SOrg & 1,039 &, 056 & 18,475 & 0,01 \\
SO6 & $<---$ & SOrg &, 863 &, 062 & 13,874 & 0,01 \\
SO5 & $<---$ & SOrg &, 998 &, 066 & 15,149 & 0,01 \\
SO4 & $<--$ & SOrg & 1,017 &, 060 & 17,070 & 0,01 \\
SO3 & $<--$ & SOrg &, 797 &, 066 & 12,061 & 0,01 \\
SO2 & $<---$ & SOrg &, 884 &, 069 & 12,876 & 0,01 \\
SO1 & $<---$ & SOrg &, 920 &, 069 & 13,367 & 0,01 \\
\hline
\end{tabular}


Vale destacar, que os indicadores de ajuste apontam, de forma robusta, para a confiabilidade do funcionamento da escala no contexto de trabalhadores de diferentes empresas no estado do Rio de Janeiro (sejam estas, públicas ou privadas). Apesar da confiança nos indicadores psicométricos, efetuou-se por fim, um teste $\mathrm{t}$ para amostras independentes a fim de avaliar as diferenças entre as pontuaçóes das respostas dos sujeitos em relação ao tipo de empresa, os quais foram as seguintes: empresas públicas [Média $=25,30, \mathrm{DP}=8,40(\mathrm{IC} 95 \%$ $=-4,37 /-2,01]$ e empresas privadas [Média $=28,49$, DP $=8,75(\mathrm{IC} 95 \%=-4,40 /-1,98]\left[\mathrm{t}_{2,633}=-5,20 ; \mathrm{p}<0,01\right]$.

Ao comparar os escores obtidos por trabalhadores de empresas públicas e privadas percebeu-se uma diferença significativa entre os dois grupos. Os trabalhadores de empresas privadas apresentaram escores significativamente superiores que os de organizaçóes públicas. Esses resultados salientam que, no presente grupo amostral, os funcionários das empresas privadas demonstraram uma maior percepção de suporte organizacional do que os servidores das organizações públicas.

\section{Discussáo}

De forma geral, pretendeu-se contribuir com este estudo para uma maior especificidade da medida de suporte organizacional em distintas empresas. Ainda que a escala tenha sido adaptada e validada por Siqueira (1995; 2008) para o Brasil, não se observou, nos estudos citados que estudaram as propriedades psicométricas do instrumento, detalhes estatísticos como análise dos componentes principais, critério de Kaiser, critério de Cattel, análise paralela e os indicadores da análise fatorial confirmatória para avaliar o comportamento dessa medida em trabalhadores.

É preciso destacar que os resultados observados no presente estudo, não devem ser considerados, simplesmente, como uma espécie de qualificação psicométrica da medida testada sobre suporte organizacional, pois, a organização da estrutura fatorial, previamente observada por autores brasileiros (Formiga et al., 2014), foi bem semelhante. Assim, o estudo contribui para garantir evidências empíricas sobre a confiabilidade e a estrutura unifatorial do instrumento para a aplicação e mensuração em outros contextos brasileiros do trabalho tanto em empresas privadas ou em públicas.

Os diversos critérios empregados para definição do número do fator a ser extraído, por exemplo, o de Kaiser, Cattell e a análise paralela (Hayton et al., 2004) reforçaram a solução unifatorial representada de acordo com o que se esperava teoricamente. A referida estrutura fatorial encontrada revelou-se adequada considerando os indicadores comumente tidos em conta para provar o modelo proposto: $\chi^{2} / \mathrm{gl}$, RMR, GFI, $A G F I, C F I$, TLI e RMSEA. Estes indicadores foram satisfatórios estando em intervalos que têm sido considerados como aceitáveis na literatura vigente sobre o assunto (Byrne, 1989; van de Vijver \& Leung, 1997).

De acordo com Siqueira (2008), ao pretender investir no processo psicológico do trabalhador em termos de seu bem-estar, faz-se necessário atentar para políticas institucionais que favoreçam esse sentimento. Neste contexto, ao salientar a importância da percepçáo de suporte organizacional, sugerese a reflexáo quanto a benefícios como horários de trabalho mais flexíveis, políticas organizacionais de apoio familiar (como creche para seus filhos), respeito às ideias dos funcionários, valorização profissional, incentivo à capacitação, acesso a informaçôes, etc., capaz de gerar maior e melhor nível de comprometimento com a organizaçáo (Oliveira-Castro et al., 1999; Dias, 1993).

Os resultados encontrados no presente estudo ratificam a qualidade do instrumento proposto por Siqueira $(1995 ; 2008)$ e corroboram os pressupostos psicométricos apontados em estudos anteriores de qualidade da medida por Formiga et al. (2014) e por Formiga e Souza (2014). Foi possível, ainda, confirmar a estrutura unidimensional do construto, o que já era apontado na literatura (Oliveira-Castro et al., 1999). Com tais evidências empíricas observadas, pode-se afirmar que o instrumento avaliado é eficaz e oferece uma medida confiável, constituindo então uma ferramenta a ser utilizada por gestores e pesquisadores da área de recursos humanos para avaliar no grupo de colaboradores a forma como os mesmos percebem o suporte oferecido pela empresa.

Apesar das evidências empíricas obtidas, algumas particularidades para futuros estudos devem ser destacadas para que se compreenda funcionamento do instrumento em diferentes situaçóes relacionadas ao trabalho: 1 - considerar uma amostra para além da especificidade da empresa pública ou privada, salientando a necessidade de distribuir a amostra por categoria de serviços; 2 - sugere-se analisar o instrumento com base no tempo de serviço, bem como, na faixa etária e retorno ao trabalho após licença de saúde; 3 - Relacionar a medida de suporte organizacional com outras variáveis que compóem o ambiente organizacional, como autoconceito profissional, produtividade, motivação, cultura organizacional, etc. 


\section{Referências}

Agapito, P. R., Polizzi Filho, A., \& Siqueira, M. M. M. (2015). Bem estar no trabalho e percepção de sucesso na carreira como antecedentes da intençáo de rotatividade. Rev. Adm. Mackenzie, 16(6), Nov/Dez.

Bisquerra, R. (1989). Métodos de investigación educativa: guía práctica. Barcelona: Ed. CEAC.

Brandão, H. P., Borges-Andrade, J. E., \& Guimarães, T. A. (2012). Desempenho organizacional e suas relaçôes com competências gerenciais, suporte organizacional e treinamento. Revista de Administração, 47(4), 523-539.

Brasil. Comissão Nacional de Ética em Pesquisa (2012). Resolução CNS no 196/96. Brasília.

Byrne, B. M. (1989). A primer of LISREL: Basic applications and programming for confirmatory factor analytic models. New York: Springer-Verlag.

Conselho Federal de Psicologia (2000). Resolução $n^{o}$ 016/2000. Dispóe sobre a realização de pesquisa em Psicologia com seres humanos. Disponível em: <http://www.crpsp.org.br/portal/orientacao/ resolucoes_cfp/fr_cfp_016-00.aspx>. Acesso em: 1 dez. 2016.

Cunha, J. A. (2000). Psicodiagnóstico V. Porto Alegre: Artmed.

Damásio, B. F. (2012). Uso da análise fatorial exploratória em Psicologia. Avaliação Psicológica, 11(2), 213-228.

Damásio, B. F. (2013). Contribuições da Análise Fatorial Confirmatória Multigrupo (AFCMG) na avaliaçáo de invariância de instrumentos psicométricos. PsicoUSF, 18(2), 211-220.

Dancey, C.P., \& Reidy, J. (2006). Estatistica sem matemática para psicologia usando SPSS para Windows. Porto Alegre: Artes Médicas.

Dias, J. M. G. (1993). Preditores do comprometimento organizacional: um estudo de caso na EMATER - MG. Dissertação de Mestrado, Universidade Federal de Minas Gerais, Belo Horizonte, Minas Gerais, Brasil.

Eisenberger, R., Huntington, R., Hutchison, S., \& Sowa, D. (1986). Perceived organizational support. Journal of Applied Psychology, 71(3), 500-507.

Fabrigar, L. R., Wegener, D. T., MacCallum, R. C., \& Strahan, E. J. (1999). Evaluating the use of exploratory factor analysis in psychological research. Psychological Methods, 4(3), 272-299.

Formiga, N., Fleury, L. F. O., \& Souza, M. A. (2014). Evidências de validade da escala de percepçáo de suporte organizacional em funcionários de empresa pública e privada. Estudos Interdisciplinares em Psicologia, 5(2), 60-76.

Formiga, N. S., \& Souza, M. A. (2014). Comprovação empírica de uma medida psicológica sobre a percepção do suporte organizacional em trabalhadores de diferentes empresas. Boletim - academia paulista de psicologia, 34(87), 510-522.

Hair, J. F., Tatham, R. L., Anderson, R. E., \& Black, W. (2005). Análise Multivariada de dados. Porto Alegre: Bookman.

Hayton, J.C., Allen, D.G. \& Scarpello,V. (2004) Factor Retention Decisions in Exploratory Factor Analysis: a Tutorial on Parallel Analysis. Organizational Research Methods, 7, 191-205.

Jawahar, I. M., \& Hemmasi, P. (2006). Perceived organizational support for women's advancement and turnover intentions: the mediating role of job and employer satisfaction. Women in Management Review, 21(8), 643-661.

Kalidass A., \& Bahron, A. (2015) ఐe Relationship between Perceived Supervisor Support, Perceived Organizational Support, Organizational Commitment and Employee Turnover Intention. International Journal of Business Administration, 6(5), 82-89.

Kelloway, E. K. (1998). Using LISREL for structural equation modeling: A researcher's guide. Thousand Oaks, CA: Sage Publications.

Ledesma, R.D., \& Valero-Mora, P. (2007). Determining the number of factors to retain in EFA: An easyto-use computer program for carrying out parallel analysis. Practical Assessment, Research \& Evaluation, 12(2).

O'connor, B.P. (2000). Behavior Research Methods. Instruments \& Computers, 32, 392-396.

Oenning, N. S. X., Carvalho, F. M, \& Lima, M. C. (2012). Indicadores de absenteísmo e diagnósticos associados às licenças médicas de trabalhadores $\mathrm{da}$ área de serviços de uma indústria de petróleo. Rev. Bras. Saúde Ocup., 37(125), 150-158.

Oliveira-Castro, G. A., Pilati, R., \& Borges-Andrade, J. E. (1999). Percepção de suporte organizacional: desenvolvimento e validação de um questionário. Revista de Administração Contemporânea, 3(2), 29-51.

Paschoal, T. (2008). Bem-estar no trabalho: relaçóes com suporte organizacional, prioridades axiológicas e oportunidades de alcance de valores pessoais no trabalho. Tese de Doutorado. Universidade de Brasília, Brasília, Distrito Federal, Brasil.

Pasquali, L. (2011). Psicometria: teoria dos testes na psicologia e na educação. 4 ed. Petrópolis/RJ: Vozes. 
Sant'Anna, A. S., \& Kilimnik, Z. M. (2011) Qualidade de vida no trabalho: abordagens e fundamentos. Rio de Janeiro: Elsevier.

Siqueira, M. M. M. (1995) Antecedentes do comportamento de cidadania organizacional: a análise de um modelo pós-cognitivo. Tese de Doutorado, Universidade de Brasília, Brasília, Brasil.

Siqueira, M. M. M. (2008). Medidas do comportamento organizacional: Ferramentas de diagnóstico e de gestão. Porto Alegre: Artmed.

Tabachnick, B. G., \& Fidell, L. S. (1996). Using multivariate statistics. Needham Heights, MA: Allyn $\&$ Bacon.

Tamayo, A., \& Paschoal, T. (2003). A relação da motivação para o trabalho com as metas do trabalhador. Revista de Administração Contemporânea, 7(4), 33-54.
Valentini, F., \& Damásio, B. F. (2016). Variância Média Extraída e Confiabilidade Composta: indicadores de precisão. Psicologia: Teoria e Pesquisa, 32(2), 1-7. Van De Vijver, F., \& Leung, K. (1997). Methods and data analysis for crosscultural research. Thousand Oaks, CA: Sage Publications.

\section{Endereço para correspondência:}

Luis Felipe de Oliveira Fleury

Rua Anna Vasco, 18

CEP: 23092620 - Campo Grande/Rio de Janeiro

Email: luis_fleury@yahoo.com.br

Recebido em 08/12/2016

Aceito em 19/01/2017 